\title{
How to be a structuralist all the way down
}

\author{
Elaine Landry
}

Received: 11 June 2008 / Accepted: 5 November 2009 / Published online: 3 December 2009

(C) The Author(s) 2009. This article is published with open access at Springerlink.com

\begin{abstract}
This paper considers the nature and role of axioms from the point of view of the current debates about the status of category theory and, in particular, in relation to the "algebraic" approach to mathematical structuralism. My aim is to show that category theory has as much to say about an algebraic consideration of meta-mathematical analyses of logical structure as it does about mathematical analyses of mathematical structure, without either requiring an assertory mathematical or meta-mathematical background theory as a "foundation", or turning meta-mathematical analyses of logical concepts into "philosophical" ones. Thus, we can use category theory to frame an interpretation of mathematics according to which we can be structuralists all the way down.
\end{abstract}

Keywords Mathematical structuralism - Category theory - Algebraic structuralism · Philosophy of mathematics · Hilbert · Frege $\cdot$ Shapiro $\cdot$ McLarty · Marquis · Hellman · Mac Lane

\section{Introduction}

This paper considers the nature and role of axioms from the point of view of the current debates about the status of category theory and, in particular, in relation to recent criticisms of the "algebraic" approach to mathematical structuralism. I first consider the Frege-Hilbert debate with the aim of distinguishing between axioms as assertions, i.e., as statements that are used to express or assert truths about a unique subject matter,

\footnotetext{
${ }^{1}$ For a precise account of what I intend by the "algebraic" approach, see Landry and Marquis (2005).
}

For John, for Mimi.

\footnotetext{
E. Landry $(\varangle)$

University of California, Davis, USA

e-mail: emlandry@ucdavis.edu
} 
and an axiom system as a schema that is used to provide "a system of conditions for what might be called a relational structure" (Bernays 1967, p. 497) so that axioms, as implicit definitions, are about whatever satisfies the conditions set forth. I then use this inquiry to rationally reconstruct those aspects of Hilbert's "foundational" programme that can be drawn on to reevaluate arguments against using category theory to frame a pure algebraic structuralist philosophy of mathematics.

With aims similar to mine, but with a decidedly different conclusion, Shapiro (2005) has claimed that the Frege-Hilbert debate can be used to show that the current algebraic structuralist debates ought to be concerned with questions that consider the status of meta-mathematical axioms (as opposed to Hellman's concerns that consider the status of mathematical axioms ${ }^{2}$ ). That is, Shapiro argues, even if we agree with the Hilbert-inspired algebraic structuralist that, at the mathematical level, "any given branch is 'about' any system that satisfies its axioms" (Shapiro 2005, p. 74), to give "criteria of acceptability" (of coherence, consistency, satisfiability, etc.) for such axioms or axiom systems themselves, we need a "foundation", as a meta-mathematical background theory, which is assertory, and so, he concludes, one cannot be a pure algebraic structuralist, i.e., a structuralist all the way down.

According to Shapiro, our only other option, as proposed by Awodey (2004) is to "kick away the foundational ladder altogether, and take the meta-mathematical settheory, structure theory, or whatever, itself to be an algebraic theory" (Shapiro 2005, p. 74). This option, however, is presented as a way not to be looked into because it supposedly has the unwanted consequence that

mathematical logic is similarly liberated from theories... our theorist can hold... that satisfiability, consistency, or coherence implies existence, but she cannot maintain that any of these notions are mathematical matters. (Ibid., 75)

The alleged result being that meta-mathematical analyses of these logical concepts are turned into non-mathematical, or, even worse, "philosophical" ones (see Shapiro 2005, pp. 74-75).

\footnotetext{
2 Hellman, for example, has argued that category theory cannot stand on its own as a "foundation" for an algebraic structuralist interpretation of mathematics because "the problem of the home address remains" (Hellman 2003, pp. 8, 15). That is, since the axioms for a category "merely tell us what it is to be a structure of a certain kind" and because "its axioms are not assertory" (Ibid., 7), we need a mathematical background theory whose axioms are assertory, i.e., a theory that assert truths about (possibly or actually) existing systems so structured. I have taken-up this criticism elsewhere and have argued that, at a mathematical level, Hellman's "home address problem" simply misses its mark. See Landry and Marquis (2005), especially footnote \#43, p. 31, where we claim "It should be underscored, however, that Hellman (2003), does appreciate the distinction between the algebraic-schematic use of categories (what he calls the 'algebraico-structuralist' perspective, p. 9), but his suggestion that the 'problem of the "home address" remains' (pp. 8,15) clearly indicates that he is still thinking of structures (be they categories or toposes) as "objects" requiring conditions for the possibility of existence. In fact, if, on the algebraic approach, the aim of structuralism is to account for the shared structure of abstract kinds of structured systems in terms of schematic types, as opposed to answering 'What is (or where is!) a structure?', then why should we be troubled by the fact that ' $[\mathrm{b}] \mathrm{y}$ themselves they [the category-theoretic axioms] assert nothing' that 'They merely tell us what it is to be a structure of a certain kind' and thus are 'unlike the axioms of set theory, [in that] its axioms are not assertory?' (p. 7)." In this paper, I consider whether, even if at the mathematical level, we resolve problems like Hellman's, we are we still left to face another, perhaps similar, set of problems at the meta-mathematical level. So my focus here is on Shapiro's most recent criticisms, though by way of dealing with these I will also offer-up a more detailed reply to Hellman.
} 
Against the criticisms and conclusion of Shapiro, my aim is to show that category theory has as much to say about a pure algebraic consideration of meta-mathematical analyses of logical structure as it does about an algebraic consideration of mathematical analyses of mathematical structure, without either requiring an assertory mathematical or meta-mathematical background theory as a "foundation", or turning meta-mathematical analyses of logical concepts into "philosophical" ones. Thus, we can use category theory to frame an interpretation of mathematics according to which we can be structuralists all the way down.

\section{The Frege-Hilbert debate}

As is well known, Frege and Hilbert debated the nature of geometric axioms. Frege held that geometric axioms are assertions; that they are statements used to express or assert truths. On the other hand, Hilbert, like our modern-day algebraic structuralist, maintained that axioms are implicit definitions. Related to these differing views of axioms, Frege and Hilbert further disagreed on at least three points. First, a theory, for Hilbert, is not a set of truths about a "fixed" subject matter. As he explains,

... every theory is only a scaffolding or schema of concepts together with their necessary relations to one another, and the basic elements can be thought of in any way one likes. If in speaking of points, I think of some systems of things, e.g., the system love, law, chimney-sweep... and then assume all my axioms as relations between these things, then my propositions, e.g., Pythagoras' theorem, are also valid for these things.... [A]ny theory can always be applied to infinitely many systems of basic elements. (Hilbert 1899, pp. 40-41)

The second point of disagreement is that, for Hilbert, a concept is fixed axiomatically, or implicitly defined, only by its relation to other concepts. It is not constructively defined $^{3}$ (in the case of Frege logically defined) by its relation to independently existing (logical) objects; rather,

... a concept can be fixed logically only by its relations to other concepts. These relations, formulated in certain statements I call axioms, thus arriving at the view that axioms .... are the definitions of concepts. (Correspondence 1900, 09/22)

The last point of disagreement is that Hilbert uses consistency to guarantee the "truth" of the axioms and hence to establish the existence of those objects that satisfy the implicitly defined concepts, and not, as for Frege, the other way round;

\footnotetext{
3 Throughout his writings, Hilbert was expressedly against both Frege and Dedekind's "construction" of the definition of numbers. For example, he characterized the method of constructing concepts as that to be undertaken via the "genetic method" and held this in sharp contrast to the method to be used to define concepts via the "axiomatic method" (See Hilbert 1900a). While it is true that he favored both methods, though for different mathematical tasks, only the axiomatic method could be put to use to (implicitly) define concepts. See Hallett (1994), p. 174, who claims "[t]he central difference [between Frege and Hilbert] is that the construction will no longer be a definition by construction, but rather only "interpretation" by construction...". Finally, see Hallett (1994) and Ewald (1999) for a more detailed explanation and discussion of this difference.
} 
[a]s long as I have been thinking, writing and lecturing on these things, I have been saying the exact reverse [of Frege]; if the arbitrarily given axioms do not contradict each other with all their consequences, then they are true and the things defined by them exist. This [consistency] is for me the criterion of truth and existence. (Correspondence 1899, 12/29)

Underlying their disagreements and debates is the fact that Frege took mathematical axioms to be assertions about independently existing objects, that is, he held that mathematics has a fixed subject matter and this is what the axioms of its branches (arithmetic and geometry) are about. As Shapiro notes:

Frege insisted that arithmetic and geometry each have a specific subject matter, space in the one case and the realm of natural numbers in the other. And the axioms express (presumably self-evident) truths about this subject matter. (Shapiro 2005, p. 67)

In contrast, Hilbert, as the precursor to the algebraic structuralist position, took the branches of mathematics (excepting, as we will see, finitary arithmetic ${ }^{4}$ ) to be about any system that satisfies its axioms ${ }^{5}$. As Bernays makes clear,

[a] main feature of Hilbert's axiomatization of geometry is that the axiomatic method is presented and practiced in the spirit of the abstract conception [the algebraic structuralist conception] of mathematics that arose at the end of the nineteenth century and which has been adopted in modern mathematics. It consists in... understanding the assertions (theorems) of the axiomatized theory in a hypothetical sense, that is, as holding true for any interpretation... for which the axioms are satisfied. Thus, an axiom system is regarded not as a system of statements about a subject matter but as a system of conditions for what might be called a relational structure". (Bernays 1967, p. 497)

Hallett, making clearer the relation between Hilbert's use of the axiomatic method and our current reconstruction of Hilbert as an algebraic structuralist, says:

In this case [in the case of the collection of Dedekind cuts exhibiting the properties that the axiom system for real number demands], axiomatization really uncovers certain structural relations that in general will be common to various structures... Indeed, the formulation of axioms then becomes one natural means of attempting to isolate structure. (Hallett 1994, p. 174)

\footnotetext{
4 As we will see, things are not so straightforward as to what "branches" Hilbert would have included in his axiomatic treatment of mathematics. As Hallett notes "[j]ust prior to this [1896] he seems to have held a version of the 'Dirichlet thesis' that all of higher analysis will in some sense 'reduce' to the theory of natural numbers, a thesis which is stated without challenge in the Vorwort to Dedekind's 1888 monograph... In the 1920s, [however] Hilbert stated decisively his rejection of the Dirichlet thesis...." (Hallett 2007, p. 34). Indeed, as early as 1918 (Ewald 1999, p. 1109), Hilbert explicitly includes arithmetic as an axiomatic theory just as any other mathematical or, indeed, scientific, theory, e.g., just as geometry, mechanics, radiation theory, or thermodynamics.

5 See Hallett (1994) for an excellent overview of Hilbert's "reference free" [variation in reference across interpretations] account of mathematics and for an explanation of how this is related to Hilbert and Frege's "differing attitudes to logic and to the laws of thought" (p. 163).
} 
We must now pause to consider the distinction between mathematics and metamathematics. For Frege, logic for arithmetic and our Kantian intuition of space for geometry is what "founds" our claims about the "truths" of their respective subject matter. Yet, even if we allow for an underlying role for set-theory in Frege's account of arithmetic, there is no obviously discernible distinction to be had between what we would now call mathematics and meta-mathematics. ${ }^{6}$

For Hilbert, by contrast, there is a clear-cut distinction. At the mathematical level, where we undertake a conceptual analysis, i.e., where we talk about the objects of various interpretations of the branches of mathematics as concepts, in terms of anything that satisfies the axioms ", no "founding", other than the organizational role afforded to the axiomatic method itself, is required. The implicit definitions of the concepts, and relative consistency or independence proofs act to guarantee the "truth" or "necessity" of the chosen axioms and, thereby establish the existence of such objects. At the metamathematical level, however, where we talk about proofs themselves as objects, we must undertake a contentual analysis, i.e., we must rely upon our intuition to "found" claims about the "truth" of those finitary arithmetical axioms which provide those "irrefutable" logical principles that underpin the underlying meta-mathematical proof theory used for an absolute consistency proof of, say, arithmetic ${ }^{8}$. That is, as Shapiro notes

[f]initary proof theory has its own unique subject matter, related to natural numbers and formal syntax, and it is ultimately founded on something in the neighbourhood of Kantian intuition. (Shapiro 2005, p. 70; italics added.) ${ }^{9}$

6 One position is that because Frege's logic is universal there is no room outside of it for metatheory (see, for example, Goldfarb 1979). The other position (see Antonelli and May 2002) is that, in light of Frege's account of geometry, where he showed how to construct independence proofs for the axioms of geometry, it is possible to give a rational reconstruction of at least some meta-logical notions.

7 For Hilbert, the interpretation, and so the objects, may be taken from any, scientific, domain of knowledge, including, for example, both mathematics and physics. As Hilbert explains, "[a]ccording to this point of view, the method of axiomatic construction of a theory presents itself as the procedure of the mapping of a domain of knowledge onto the framework of concepts, which is carried out in such a way that to the objects of the domain of knowledge there now corresponds the concepts, and to the statements about the objects there corresponds the logical relations between concepts" (Hilbert 1921/1922 in Hallett (2007), p. 9).

8 So the "truths" of finitary arithmetic, for Hilbert, are not true in the Fregean sense of being about a unique subject matter but rather are true in the Kantian a priori sense [see Hilbert 1931a, 1930b in Ewald (1999)] that the intuitive operations and principles that give rise to the axioms for finitary arithmetic/proof theory are irrefutable because they are founded on (Kantian) preconditions for (pure) reasoning itself. That is, the intuitive operations and principles that underlie our symbolic reasoning about natural numbers as signs, i.e., as sequences of strokes, give rise to the logical structure of the axioms of finitary arithmetic and these to those logical principles that underlie our symbolic reasoning about formulas and formal proofs as signs. This precondition, as Zach explains, is such that "[i]n order to carry out the task of providing a secure foundation for infinitary mathematics, access to finitary object [as signs] must be immediate and certain". (Zach 2006, p. 423) But it was the resulting proof-theoretic formalism and not the contentual reasoning, nor the "philosophy" underlying it, which did the meta-mathematical work. So, for example, as Zach notes "Hilbert and Bernays developed the $\varepsilon$-calculus as their definitive formalism for axioms systems for arithmetic and analysis, and the so-called $\varepsilon$-substitution method as the preferred approach to giving consistency proofs" (Ibid., 417).

9 As I will show, what is at stake here is just what is meant by the term 'founded'. It suffices to point out here, however, that, as explained in the endnote above, it is not intuition that founds the subject matter of proof theory; it is the "irrefutability" of finitistic reasoning about signs that does the founding as a 


\section{Shapiro's criticisms and conclusion}

As noted above in my introductory remarks, Shapiro's recent criticism of the pure algebraic structuralist's use of category theory appeals to elements of this sketch of the Hilbert-Frege debate to point out that even if, at the mathematical level, category theory can be used to argue for an algebraic account of mathematics, where the category-theoretic axioms act as implicit definitions of the concepts of mathematics, at the meta-mathematical level, the category-theoretic axioms, themselves, need to be assertions. According to Shapiro, the category-theoretic pure algebraic structuralist, if he is to avoid the "philosophical" pitfall similar to that faced by Hilbert, is forced to "found" his meta-mathematical category-theoretic axioms on some assertory theory, i.e., on some theory that asserts truths and, in so doing, can "answer legitimate foundational questions" (Shapiro 2005, p. 71).

More pointedly, Shapiro's claim is that meta-mathematical analyses of those logical concepts used as "criteria of acceptability", like coherence, consistency, satisfiability, etc., themselves require "founding" by an assertory theory of sets or structures. This allegedly implying the conclusion that we must either accept that we cannot be pure algebraic structuralists, i.e., structuralists all the way down, and so must go "foundational" (by accepting an assertory meta-mathematical background theory) or we must reject foundations and, like Hilbert's appeal to Kantian intuition, go "philosophical". My aim, then, is to show, at least in the category-theoretic case, this dichotomy is false.

Shapiro first presents us with the three "foundational" options that can be used to "save" the algebraic structuralist: Hellman's modal set theory, Shapiro's modeltheoretically motivated structure theory, or McLarty's category theory, as framed by either the ETCS (Elementary Theory of the Category of Sets) or CCAF (Category of Categories as a Foundation $)^{10}$ axioms. Shapiro next claims that, to fulfill its "foundational" role of meta-mathematically analysing those logical criteria of acceptability, each must be taken as an assertory meta-mathematical background theory;

[t]o be sure, if a category-based theory is to play this role, then its axioms must be assertory...each of them (the category based set theory, modal set theory, structure theory) is not just another mathematical theory, providing an implicit definition of some structures, or isomorphism types. The reason for this is that .... [each] has a foundational role to play concerning the coherence of definitions. And this last is an assertory matter. (Shapiro 2005, pp. 73-74)

Shapiro then notes that, in contrast to the above "foundational" options, for category-theoretically minded structuralists, there is also the purely algebraic,

\footnotetext{
Footnote 9 continued

precondition for reasoning. That is, 'intuition' is used here not in the Kantian-Fregean sense, as in intuition of space or time, i.e., as a precondition for the construction of concepts; rather, it is used in the Kantian sense as a precondition for pure thought itself. As Hilbert explains, "The a priori is here nothing more and nothing less than a fundamental mode of thought, which I also call the finite mode of thought... (Hilbert 1931a, in Ewald (1999), p. 1150)

10 For a more precise, formal, account of the ETCS axioms see, for example, Lawvere (1964) and Mac Lane (1986). For a more precise, formal, account of the CCAF axioms, see, for example, Lawvere (1966) and McLarty (1991).
} 
"non-foundational", alternative. This option is claimed by Shapiro to be in line with Awodey (2004) where we

...kick away the foundational ladder altogether, and take the meta-mathematical set-theory, structure theory, or whatever, itself to be an algebraic theory. On this view, set theory does not directly serve as a court of appeal for matters of coherence and thus existence.... The axioms of set theory are just implicit definitions that, if coherent, characterize a structure or a class of structures. The same goes for structure theory, modal set theory, and the various topos theories. (Shapiro 2005, p. 74)

The problem, as Shapiro sees it, is that

[o]n this view, everything in mathematics is algebraic. So if there is to be an assertory canonical backdrop - a non-algebraic theory of coherence, consistency, mathematical existence, whatever - it will be relegated outside of mathematics, perhaps to philosophy... [otherwise] we will go back to the plan executed in Hilbert's Grundlagen, and settle for the analogue of relative consistency proofs. (Shapiro 2005, p. 74)

But oddly, while considering the possibility of our having to "settle" for relative consistency proofs, Shapiro continues on to give us the tools for the construction of his own demise. He first says of the "second theoretical option", which includes Awodey's, purely algebraic, non-foundational account:

[n]otice that we have no formal assurance that our [background theory] is itself coherent... (Shapiro 2005, p. 74),

but then goes on to say of his own model/set-theoretically motivated structure-theoretic foundational account

[o]n the first theoretical option, where the meta-theory is assertory, we likewise have no theoretical assurance that set theory is true. Again we have no safety net, and do not really need one. (Shapiro 2005, p. 74)

We are here left asking: Why is it that the advocate of the "foundational" option needs no assurance that this background meta-mathematical theory is true, yet the proponent of "non-foundational" option is required to show that his background theory is coherent/consistent? Surely, given their difference of opinion as to the nature of axioms of their chosen theories (respectively, as either assertions or as implicit definitions), these "acceptability problems" are either equally pointed (the axiomsas-assertions foundationalist faces the "truth problem" to the same extent that the axioms-as-implicit-definitions non-foundationalist the "coherence/consistency problem") or they equally dissolve (the non-foundationalist can likewise claim that he does not have, or need, a "safety net").

Before continuing on to make the case for category theory, I have three things to note. First, Shapiro's structure theory, even if cast in the frame of the axioms for ZF 
(see Shapiro $1997^{11}$ ), appears to be more "philosophical" than any category-theoretic option; structure-theory is clearly not either a mathematical or a meta-mathematical theory. Second, Shapiro himself has been far more concerned with the "coherence" of his structure theory axioms than with their truth; even going as far as to include a "coherence axiom" (again, see Shapiro 1997). So, unless he further provides arguments for a coherentist account of truth, he has no quick route from coherence to truth. Finally, and perhaps most problematic, Shapiro has, in making the categorytheoretic "foundational" versus "non-foundational" distinction, conflated at least two, if not three, category-theoretic levels. That is, even if the ETCS axioms are claimed to "found" branches of mathematics that are organized set-theoretically, it is only the CCAF axioms that are claimed to "found" category theory.

\section{The case for category-theory}

To understand what is at issue here, I begin with an abstract definition of a category.

Definition A cat-structured system $C$ (a category) is an abstract system of two abstract kinds; objects $X, Y, \ldots$ and morphisms $f, g, \ldots$ such that

\section{Eilenberg-Mac Lane (EM) axioms:}

(a) Each morphism $f$ has an object $X$ as a domain and an object $Y$ as a codomain, indicated by writing $f: X \rightarrow Y$.

(b) If $g$ is any morphism $g: Y \rightarrow Z$ with domain $Y$ (the codomain of $f$ ) and codomain $Z$, there is a morphism $h=g \circ f$ called the composition of $f$ and $g$.

(c) For each object $X$ there is a morphism $1_{x}: X \rightarrow X$ called the identity morphism of $X$.

(d) These objects and morphisms satisfy:

(i) Associativity: $f \mathrm{o}(g \mathrm{o} h)=(f \circ g) \mathrm{o} h$

(ii) Identity: For all $X$ the domain of $1_{x}=$ codomain of $1_{x}=X$ and for all $f$, $f \circ 1_{x}=f, 1_{y} \circ f=f$

The claim of the category-theoretic algebraic structuralist is that the above cat-structured system acts as an abstract Hilbertian axiom system ${ }^{12}$; it provides an abstract schema for organizing the mathematical structure of both the concepts of the branches of mathematics and the concept of a category itself.

In the former case, for example, the following categories allow us to organize the mathematical structure of the concepts set, group, topological space, etc.

11 In this regard Shapiro himself claims: "[m]y own structure theory (Shapiro [1997], Chap. 3) was meant to play the same assertory, foundational, role as set theory, and, indeed, structure theory is a notation variant of set theory" (Shapiro 2005, p. 73).

12 As Mac Lane explains: [i]n this description of a category, one can regard "object", "morphism", "domain", "codomain", and "composites" as undefined terms or predicates (Mac Lane 1968, p. 287; italics added). 
Set - where we take sets as objects, functions as morphisms,

Grp-where we take groups as objects, homomorphisms as morphisms,

Top-where we take topological spaces as objects, continuous functions as morphisms,

Diff — where we take differential manifolds as objects, smooth maps as morphisms,

Lat and Bool—where we take lattices and Boolean algebras as objects, respectively, and $(\top, \perp, \wedge, \vee)$ homomorphisms, as morphisms

Heyt - where we take Heyting algebras as objects and $(\top, \perp, \wedge, \vee, \rightarrow)$ homomorphisms as morphisms

Rings - where we take rings as objects and ring homomorphisms, i.e., $(0,1,+, \times)$ homomorphisms, as morphisms. ${ }^{13}$

The ETCS axioms, begin with the above abstract Eilenberg-Mac Lane (EM) axioms and applies them to sets as objects and functions as morphisms, so that the axioms are satisfied; for example, every function $f$ goes from a unique set $X$ to a unique set $Y$, every set $X$ has an identity function, etc. Thus the ETCS axioms, as the ZF axioms, can be used to analyse the mathematical or logical structure of concepts that are organized set-theoretically ${ }^{14}$ (except, of course, the category of all sets, SET, of all groups, GRP etc. ${ }^{15}$ )

More pointedly, the ETCS axioms, as Shapiro intends of set- or structure-axioms, can be used to meta-mathematically analyse those logical concepts (consistency, satisfiability, independence) used as "criteria of acceptability" for axiom systems themselves. To talk about the mathematical structure of categories themselves, including the meta-mathematical structure of the category Set of all sets ${ }^{16}$ as framed by the ETCS axioms, we can use the CCAF axioms; where now, in the abstract definition above, categories are objects and functors are morphisms. In so doing, we use the CCAF axioms ${ }^{17}$ as a Hilbertian axiom system or schema for the concept of a category itself. Thus, we have a means for both talking about the meta-mathematical structure of the concepts of the branches of mathematics that are organized in category-theoretic terms, Set included, and for meta-mathematically analysing the concept of a category itself in terms of the structure of Cat (again, except for the category of all categories, CAT).

We must now turn to ask: What "foundational" role are the category axioms, both those of ETCS and of CCAF, intended to play and what is meant by 'foundation'? To answer these questions, I refer to the writings of McLarty (2005) who notes that there

\footnotetext{
13 These examples are taken from Marquis (2007) which provides a more detailed list of categories for various mathematical concepts.

14 Basically, the ETCS axioms plus an adjoined axiom scheme of replacement yields a set theory equivalent to ZF. See McLarty (2007) for more details.

15 There are ways, of course, as there are for the set-theorist, of bypassing these "size" problems; for example, by appealing to Grothendieck universes. As McLarty notes: "[o]n ZF foundations a Grothendieck universe is a set satisfying all the ZF axioms. In ETCS foundations it is a set of sets which, together with all the functions between them, satisfy the ETCS axioms. Either way a Grothendieck universe proves the consistency of its set theory, so that neither ZF nor ETCS proves there are universes" (McLarty 2007, p. 11).

16 This because the CCAF axioms prove a theorem scheme of unbounded set for Set; again see McLarty (2007) for details.

17 See McLarty (2007), especially pp. 13-18 for details of the CCAF axioms.
} 
are two senses of the term 'foundation', arising from two uses of 'axioms' ${ }^{18}$ In the first, Aristotelian sense, an axiom (whether it is an assertion or an implicit definition) is that which itself must not admit of any proof. In the second sense, an axiom is that which "must be independently plausible to a reasonably sophisticated mathematician" (Ibid., 44) ${ }^{19}$. Resulting, then, from the first sense of 'axiom', a foundation must account for the privileged status of such "proof-less" axioms.

By contrast, and in line with the second sense of 'axiom', a foundation, as considered by Mac Lane (1986) for example, can be seen as a proposal for the structural organization of mathematics via the axiomatic method ${ }^{20}$. Further witnessing the Hilbertian heritage of the resulting category-theoretic structuralist consideration of an axiom system qua relational structure, Mac Lane states:

... a structure is essentially a list of operations and relations and their required properties, commonly given as axioms, and often so formulated as to be properties shared by a number of possibly quite different specific mathematical objects... a mathematical object 'has' a particular structure when specified aspects of the objects satisfy the (standard) list of axioms for the structure. This notion of 'structure' is clearly an outgrowth of the widespread use of the axiomatic method in mathematics [as exemplified by Hilbert's Grundlagen]. (Mac Lane 1996, pp. 174, 176)

It is in this second sense, then, that McLarty suggests that category theory is to be taken as a foundation and adds that, even if it is not the last word, "[i]t is the latest and currently best word in the structuralist organization of mathematics" (McLarty 2005, p. 45). More to the point, at the mathematical level, i.e., at the level where we consider the organization of categories themselves, it is only the CCAF axioms, and not either the ETCS (as Shapiro claims might be the case for McLarty) or the EM axioms (as the Awodey path suggests) that are used to respond to Hellman (2003) by showing

18 See McLarty (2005), p. 44, footnote \#3: "I follow the line Saunders Mac Lane often takes ([1986], p. 406), whereby foundations are 'proposals for the organization of Mathematics', which I believe is much like what Shapiro [1991] means by 'foundations without foundationalism'. To count as a foundation the axioms must be independently plausible to a reasonable sophisticated mathematician".

19 My aim (see p. 16) will be to provide a middle-ground between the Aristotelian "proof-less" account of an axiom and McLarty's "plausibility" account, which, in appealing to what is "independently plausible to a reasonably sophisticated mathematician", seems to allow for a socially constructed component that is both not wanted and not warranted. The idea here will be to show that an axiom, or axiom system, is taken as "plausible" in service of its foundational role, that is, because it structurally organizes concepts. There are, of course, logical criteria of acceptability which, thought these criteria are themselves formal, are nonetheless variable, e.g., they may be chosen from a semantic, syntactic or finitistic perspective and for various tasks, like organizing constructive mathematics; in any case, they are not either social or, to borrow Shapiro's term, "philosophical" criteria. As Marquis notes, in the category-theoretic setting, an axiom or axiom system is "plausible" to the extent that "it is what is basically required for a conceptual framework to work in the way in should... For instance, a homology theory, as axiomatized by Eilenberg and Steenrod, is basically any functor between two categories satisfying their axioms. It is not that the axioms are true, not that they are coherent (we already know they are in this case), but they specify norms for such a theory to qualify as a homology theory and these norms are basic properties found in all homology theories." (Marquis, personal correspondence)

20 Note, however, that Mac Lane himself was no mathematical structuralist. See Landry and Marquis (2005) for a more detailed account of Mac Lane's position. 
that category theory requires no "home address". ${ }^{21}$ Simply, the CCAF axioms as an axiom system qua Hilbertian schema for the structural organization of the concept of a category itself, are not assertory. So McLarty concedes to Hellman the point that

[the Awodey way] is a fine way to work for some purposes [for abstractly organizing concepts in category-theoretic terms] but Hellman is right that we also have foundational concerns [for organizing the concept of a category itself]. When we pursue these we cannot be satisfied with Awodey's equation, where he says 'the question of whether the conditions [for any given theorem] are ever satisfied' is just the question of 'whether they [the EM axioms] are consistent'. (McLarty 2005, p. 53)

Yet, McLarty further clarifies, and, in so doing, breaks the (false) dichotomy between the first "foundational" option and the second "non-foundational" option, at least as regards the category theorist. That is, McLarty responds directly to Hellman's concerns, without, as does Shapiro, conflating the category-theoretic levels and the respective roles played by the CCAF and ETCS axioms, and without, as Shapiro does by confusedly placing McLarty's position amongst those adopting the first option $^{22}$, taking the CCAF axioms as assertory:

[t]he key point to grasp here is precisely that categorical foundations for category theory are not set-theoretical foundations for category theory. When we axiomatize a meta-category of categories, by the axioms of CCAF, the categories are not 'anything satisfying the algebraic axioms of category theory'-i.e., the Eilenberg-Mac Lane axioms. They are anything whose existence follows from the CCAF axioms. They are precisely not sets satisfying the Eilenberg axioms. They are categories as described by Lawvere's CCAF axioms. (McLarty 2005, p. 52; italics added.)

\footnotetext{
21 As McLarty points out elsewhere, the question of the existence of categories is not a question of whether its axioms are assertory: "Indeed category theory per se has no such [assertory] axioms, but that is no lack, since category theory per se is a general theory applicable to many structures. Each specific categorical foundation offers various quite strong existence axioms" (McLarty 2004, p. 43).

22 Witnessing this conflation and the resulting confusion, I point the reader to Shapiro's claim that "McLarty [2004] argues that a set theory formulated in categorical terms, such as ETCS or CCAF, will work even better as a canonical backdrop than the more standard Zermelo-Fraenkel set theory. To be sure, if a category-based theory is to play this role, then its axioms must be assertory... This is not to say that McLarty himself takes the axioms of some category-based set theory this way. His point is that if one needs or wants a set theory to serve as a canonical backdrop for questions of existence, as on our first option, then a category-based set theory will do the job as well as, or better than, the more standard Zermelo-Fraenkel set theory" (Shapiro 2005, p. 73). First, I note that McLarty (2004) does not argue that the CCAF axioms can play this set-theoretic "canonical backdrop" role and that even if the ETCS axioms can play this set-theoretic role they are not intended, as are the CCAF axioms, as a canonical backdrop for questions of existence of categories. In any case, I will show that this does not mean, even for McLarty, that the ETCS axioms must be taken as assertory. Second, I will also show, again even for McLarty, that the CCAF axioms, in their foundational role as providing a "canonical backdrop" for the concept of a category itself, i.e., for questions of the existence of categories, are not taken as assertory either. So it is simply a mistake to conclude, as Shapiro does, "that set theory, structure theory, [category theory], etc., has a foundational role to play... And this last is an assertory matter." (Ibid., 74)
} 
The CCAF axioms, then, are intended to be foundational in both a mathematical and meta-mathematical, yet non-assertory, sense, i.e., in the sense that they organize what we say about the implicitly defined concept of category itself, and in the sense that they are about any object that $i$ a category, including the category Set as organized the ETCS axioms. Yet too they are foundational not in the Aristotelian sense that they are accepted because they account for the claim that the axioms are privileged or "proof-less" but in the sense that they are accepted because they are organizational of both the mathematical structure of categories and the meta-mathematical structure of anything that is a category. Thus, we can clearly use the CCAF axioms to respond to Hellman's concerns without, as Shapiro claims, our having to take either the CCAF or the ETCS axioms as assertory. The problem, for the pure algebraic structuralist (McLarty included), with Awodey's scheme is not that he takes the axioms as non-assertory but rather that he takes the EM axioms, as opposed to the CCAF axioms, to be the axioms that implicitly define categories.

The question that remains, however, is whether we can give a category-theoretic account of meta-mathematical analyses of those logical criteria of acceptability that does not rely, as McLarty suggests, on the vague, and possibly socially constructed, notion of what is "plausible to a reasonably sophisticated mathematician" and too that does not require, as Shapiro claims, either a "foundation", as an assertory metamathematical background theory, or our "turning to philosophy". To make the above situation more perspicuous, and, in so doing, set the stage for my response to Shapiro's criticisms, let us return to perhaps glean more from a more detailed algebraic reconstruction of Hilbert's "foundational" programme.

\section{An algebraic reconstruction of Hilbert}

Hilbert too can be seen as having made the distinction between using 'axiom', and so 'foundational', in the organizational versus the Aristotelian sense. Reconstructing Hilbert along these lines, we see two distinct components of Hilbert's "foundational" programme; the mathematical project of founding, in the organizational sense, mathematics (indeed, and all scientific thought) on the axiomatic method ${ }^{23}$ and the meta-mathematical project of founding, in the Aristotelian sense, the axioms of infinitary arithmetic and proof theory by finitary, intuitive, means. That is, this meta-mathematical founding was needed so that the axioms of finitary arithmetic could be taken as privileged (as "irrefutable", and so as not requiring proof) with the result that proofs themselves can be taken as "objects" of logical analysis to be then used to prove, by finitary means, the absolute consistency of infinitary arithmetic. Merging these components with those that form the basis of Shapiro's criticisms

\footnotetext{
23 In addition to footenote 7, and further witnessing this "organizational" aspect, see, for example, Hilbert's claim that "[e]very science takes its starting point from a sufficiently coherent body of facts as given. It takes form, however, only by organizing this body of facts. This organization takes place though the axiomatic method, i.e., one constructs a logical structure of concepts so that the relationship between the concepts corresponds to the relationship between the facts to be organized. There is an arbitrariness in the construction of such a structure of concepts; we, however, demand of it: (1) completeness, (2) independence, (3) consistency." (Hilbert 1902 in Hallett and Majer 2004)
} 
we come to consider three aspects of Hilbert's "foundational" programme, i.e., the conceptual, logical and meta-mathematical, which can now be put to use to reconstruct some of the finer details of Hilbert's algebraic structuralism:

(1) When conceptually analysing the mathematical structure of a given branch of mathematics, we have axioms as implicitly defining concepts; here our task is to present an axiom system qua conceptual schema for the facts of any given interpretation (which provides a domain of objects for these concepts) in such a way as to organize what can be mathematically asserted about such objects as concepts, i.e., what can be asserted in terms of anything that satisfies the axioms. ${ }^{24}$

(2) When logically analysing axioms or axiom systems of specific concepts, we make use of logical criteria of acceptability, e.g., completeness, independence and consistency; here our task is to give an account of those axioms that are necessary 25 and prove the consistency of these axioms relative to, for example, the theory of arithmetic, and thereby establish the existence of those objects that satisfy the implicitly defined concepts.

Having undertaken both (1) and (2) for the branches of mathematics, we thereby establish, via the axiomatic method, a conceptual foundation for mathematics, where 'foundation' is taken in the organizational sense of the term.

Turning next to Hilbert's meta-mathematical project, we have:

(3) When meta-mathematically analysing the logical structure of proofs themselves we take proofs as objects (as finitely intuited signs) and undertake a contentual analysis of their logical structure, i.e., we "turn to philosophy" and rely on our (Kantian) intuition to "found" claims about the "truth" of those finitary arithmetical axioms which provide "irrefutable" logical principles that underpin the underlying meta-mathematical proof theory used for an absolute consistency proof of, say, arithmetic itself.

Having undertaken (3) we thereby establish, via an appeal to Kantian intuition, a contentual foundation for mathematics and for mathematical logic/mathematical reasoning, i.e., a secure foundation for (finitary) mathematics from which we can

\footnotetext{
24 Where what can be asserted is no longer dependent upon the intuitive construction of concepts, i.e., constructions made on the basis of either our Kantian intuition of space or time, as it was thought to for Frege's conception of geometry or Peano's conception of arithmetic, or on the logical construction of concepts, as was both Dedekind and Frege's construction of the concept of number and Russell and Whitehead's construction of the concept of set.

25 See Hallett (2007) for a detailed discussion of the search for the "necessary" axioms of geometry as an example of Hilbert's attempt to reach an epistemological "purity of method", a method equally free from both intuitive assumptions (giving rise to the mistaken belief in the "truth" of the parallel axiom) and analytic assumptions (giving rise to the mistaken belief in the "truth" of the continuity axiom), so that an axiom system, as a framework for concepts, when reduced to its necessary axioms could then be used to conceptually organize mathematics, and, indeed, all of scientific thought. So by 'necessary' is meant needed to frame what we can say about all possible interpretations. As Hilbert explains: "Nevertheless [in spite of it being free of a particular interpretation] this framework of concepts has a meaning for knowledge of the actual world, because it represents a 'possible form in which things are actually connected'. It is the task of mathematics to develop such conceptual frameworks in a logical way, be it that one is led to them by experience or by systematic speculation" (Hilbert 1921/1922 in Hallett and Majer 2004).
} 
securely extend, by a proof of absolute consistency, to infinitary mathematics, where 'foundation' is here taken in the Aristotelian sense.

So, at the mathematical level, when undertaking a conceptual analysis of mathematical structure, Hilbert was happy to let the axioms speak for themselves, modulo the logical analysis of certain "criteria of acceptability". At the meta-mathematical level, when undertaking a contentual analysis of logical structure, he "turned to philosophy" 26 and used intuition to further "found" the "truth" (privileged and "proof-less" status) of those "logical principles" underpinning the logical structure of the proof-theoretic axioms. This with the aim of both showing that the axiomatic method applies to logic itself ${ }^{27}$ and of providing a "natural" 28 account of infinitary arithmetic, analysis, set-theory, etc. ${ }^{29}$

\section{Responding to Shapiro}

Thus, accepting (as Shapiro does) the lesson of Gödel, that proofs of absolute consistency by finitary means is a way not to be looked into, there are nonetheless three ways the category-theoretic pure algebraic structuralist can use this rational reconstruction of Hilbert to respond to Shapiro's meta-mathematical challenge, and none requires our taking category-theoretic axioms as assertions or our "turning to philosophy". These are:

(1) When conceptually analysing the abstract mathematical structure of the concepts of any given branch of mathematics, we have the EM axioms as implicitly defining the abstract concept of a category; here our task is to present an axiom system qua an abstract conceptual schema for the facts of any given interpretation (which provides a domain of objects, i.e., 'objects' and 'morphisms', for

\footnotetext{
26 I remind the reader to consider my claim that Hilbert "turned to philosophy" in the light of what I said in footnotes 8 and 9 . That is, it is merely for the sake of argument with Shapiro that I concede (unwillingly) to this claim. Moreover, I take the examples on pages 23 and 24 to show that rationally reconstructing Hilbert along category-theoretic lines shows that the pure algebraic structuralist does not have to make any such "turn". See also footnote 34.

27 See Hilbert 1930b in Ewald (1999), especially, p. 1159)

28 That is, an account that would "do full justice to the constructive tendencies, to the extent that they are natural" (Hilbert 1922a in Ewald 1999, p. 1119) and in so doing avoid the "unnatural" and problematic accounts of Kronecker and his followers, Weyl and Brouwer.

29 Note then that his aim was not to secure any one theory, arithmetic for example, as a foundation, but rather his goal with his "new [proof-theoretic] grounding of mathematics" was to "rid the world of the question of the foundations of mathematics once and for all by making every mathematical statement into a formula that can be concretely exhibited and rigorously derived, and thereby bring mathematical conceptformations and inferences into such a form that they are irrefutable and yet furnish a model of the entire science". (Hilbert 1931a, in Ewald (1999), p. 1152) That is, by showing that mathematical thought "takes place parallel to speaking and writing; by the formation and placing together of sentences. And for justification I need neither God, like Kronecker, nor the assumption of a special capacity of our understanding directed towards the principle of complete induction, like Poincaré, nor some ur-intuition like Brouwer, nor, like Whitehead and Russell, the axioms of infinity and reducibility, which are real, contentual presuppositions, not compensated by proofs of consistency, and of which the latter is not even plausible..." (Hilbert 1930b, in Ewald (1999), p. 1157). Hilbert claims to have "fully attained what I desired and promised: The world has thereby been rid, once and for all, of the question of the foundations of mathematics as such" (Ibid.)
} 
these concepts) in such a way as to organize what can be mathematically asserted about such objects as abstract, cat-structured, concepts, i.e., what can be asserted in terms of anything that satisfies the EM axioms

i. When conceptually analysing the concepts of the branches of mathematics that are themselves organized set-theoretically, the category theorist can take the ETCS axioms as a mathematical conceptual scheme for organizing, in categorytheoretic terms, what we say about the mathematical or logical structure of these set-structured objects as, cat-structured, concepts, i.e., what can be asserted in terms of anything that satisfies the ETCS axioms.

ii. When conceptually analysing the concept of a category itself, the category theorist can take the CCAF axioms as both a mathematical and a meta-mathematical conceptual scheme; respectively, in the sense that they organize what we say about the concept category itself, and in the sense that are about any object that is a category, including the category Set, as organized by the ETCS axioms, i.e., what can be asserted in terms of anything that satisfies the CCAF axioms.

(2) When logically analysing axioms or axiom systems themselves, either at the abstract (EM), mathematical/set-structured (ETCS) or meta-mathematical/catstructured (CCAF) level, the category theorist can make use of the resources of the many categorical logics ${ }^{30}$ to organize what we say about those logical concepts, like completeness, independence, consistency, coherence, satisfiability, etc, that are used as "acceptability criteria" for axioms or axioms systems themselves. Here our task is, again, to give an account of those axioms that are necessary and prove, for example, the consistency of these axioms relative to some stronger ${ }^{31}$ theory, and thereby establish the existence of those objects that satisfy the implicitly defined concepts.

Having undertaken both (1) and (2) for the branches of mathematics, category theory included, we thereby establish, via the axiomatic method, a conceptual foundation for mathematics, where 'foundation' is taken in the organizational sense of the term.

We are still left to face the charge that, at the meta-mathematical, now contentual, level, we, like Hilbert, must "turn to philosophy". That is, it is with respect to our reconstructed third way that Shapiro believes that he has one more sword to swing at the category-theoretic pure algebraic structuralist. As noted, Hilbert's metamathematical, proof-theoretic, analysis takes proofs themselves as objects; it is concerned not with the conceptual analysis of the logical relations that bear between concepts, like sets, groups, etc., but rather with the contentual analysis of logical relations that bear between proofs themselves. Thus, according to Shapiro, even if we do not kick away the foundational ladder and so maintain category theory, as defined by the CCAF axioms, as a meta-mathematical foundation, now, however, in the organizational sense of the term, we are we still left to face the charge that at the meta-mathematical, contentual, level we like Hilbert, must "turn to philosophy":

30 See Marquis (2007) for a more detailed description of categorical logic and for an extensive list of references; see especially the section entitled "Research papers on various aspects of categorical logic".

31 For example, CCAF is consistent if ZFC and one Grothendieck universe is. 
[a]s a structuralist, our theorist can hold -in assertory philosophy- that satisfiability, consistency, or coherence implies existence, but she cannot maintain that any of these notions are mathematical matters. There are simply no distinctly mathematical objects, and so theories, deductions, and interpretations are not mathematical. But perhaps we should not quibble over labels. (Shapiro 2005, p. 75; italics added.)

In response, I note that there is nothing to quibble about. Regardless of labels, on the category-theoretic pure algebraic structuralist view, a meta-mathematical analysis of the content (semantic, syntactic, or finitistic) of the logical structure of the concepts of mathematical logic/mathematical reasoning, including statements of consistency, coherence, etc., does not require a non-mathematical, "philosophical", analysis.

Indeed, as Marquis claims, "this is the very first moral: the distinction between mathematics and meta-mathematics more or less evaporates in a category-theoretical framework" (Marquis, personal correspondence) ${ }^{32}$. More pointedly, when considering a meta-mathematical semantic analysis of the various model-theoretic concepts of satisfiability, interpretation, truth, relative consistency, etc., as Marquis explains,

it is easy to define these notions in the appropriate categories and these are nothing more than a generalization of Tarski's notions. With a bonus: it is easy and natural to do this for multi-sorted languages...Standard references: Makkai and Reyes (1977), Johnstone (2002). (Marquis, personal correspondence)

So, the 'sorts' need not be taken as sets or structures, though of course, they could be taken as sets; in which case, as noted, we can use the ETCS axioms to provide a meta-mathematical analysis of the semantic content of the various model-theoretic concepts of satisfiability, interpretation, truth, relative consistency, in so far as we consider these concepts themselves as organized set-theoretically.

And too, in line with Hilbert's meta-mathematical, proof-theoretic, analysis of syntactic content, category theory allows us to describe deductive systems in terms of categories, so we can employ categorical methods for proof-theoretic purposes. For example, one can analyse the proof-theoretic structure of any system by using Ded, the category of deductive systems, which takes 'objects' as formulas, 'morphisms' as proofs or deductions, and operations on morphisms as rules of inference (See Lambek and Scott 1986). ${ }^{33}$

Finally, in line with Hilbert's preference for finitistic reasoning, we can use topos theory to meta-mathematically analyse the finitistic content of the various

\footnotetext{
32 As Marquis (2007) explains, the reason that there is no distinction between mathematics and meta-mathematics is that the resources of the various categorical logics can be used to analyze logical concepts as considered from within those systems that are organized by either the EM, the ETCS or the CCAF axioms.

33 As Marquis (2007) notes "It is therefore legitimate to think of a category as an algebraic encoding of a deductive system. This phenomenon is already well-known to logicians, but probably not to its fullest extent. An example of such an algebraic encoding is the Lindenbaum-Tarski algebra, a Boolean algebra corresponding to classical propositional logic. Since a Boolean algebra is a poset, it is also a category... Thus far we have merely a change of vocabulary. Things become more interesting when first-order and higher-order logics are considered."
} 
aspects of constructive mathematics, including constructive set theory, the concepts of recursiveness, independence, and models of higher-order type theories generally. ${ }^{34}$ Thus, one can appeal to category-theoretically organized concepts, axiom systems and methods to meta-mathematically analyse the content (semantic, syntactic, or finitistic) of the logical structure of mathematical logic/mathematical reasoning itself, including the content of statements of consistency, coherence, etc.

Clearly, then, Shapiro is mistaken in his claim that meta-mathematical analyses of those logical concepts used as "criteria of acceptability", like coherence, consistency, satisfiability, deductive system, etc., themselves require "founding" by an assertory meta-mathematical background theory. And so the category-theoretic structuralist is in a position to reject his conclusion that we must either accept that we cannot be algebraic structuralists all the way down and go "foundational" (by accepting an assertory meta-mathematical background theory) or we must accept Aristotelian foundations and, like Hilbert's appeal to Kantian intuition, " turn to philosophy" ${ }^{35}$ I have shown we need not do either. This because statements of the consistency, coherence, etc., of some mathematical theory are assertory in the theory (for example, in the meta-mathematical theory as structured by some categorical logic) that is taken as stronger for the proof of the relative consistency/coherence of the theory in question. This, however, does not mean that the stronger theory itself must be true, i.e., that its axioms must be assertory, it means it must be strong enough ${ }^{36}$ to assert that the theory in question is consistent/coherent. ${ }^{37}$

\footnotetext{
34 Again, as Marquis explains “... Hilbert's [finitary] program is getting new fuel from categorical logic! There is some fascinating work done by mathematicians on constructive proofs of classical results using at their core geometric logic and basic theorems of preservation in the topos-theoretical setting. This is a beautiful example of what can be done in this framework". (Marquis, personal correspondence). See Marquis (2007) for a detailed list of references and for a brief sketch of the history and current uses of topos theory. For more on the history of topos theory, see McLarty (1992).

35 Note, however, that there are several rational reconstructions of the finitistic aspect of Hilbert's programme that are mathematical, so that, against Shapiro's claim, even for Hilbert, "going philosophical" need not be the only alternative to "going foundational/assertory". Here I have in mind Tait's (1981) claim that finitistic reasoning is just primitive recursive reasoning, so that appeals to Kantian intuition can be dispensed with. See Zach (2006), however, for criticisms of this view and for the presentation of various other alternatives, both mathematical, e.g., Kreisel's (1960), and philosophical, e.g., Parson's (1998).

36 I mean whatever, in light of Gödel results, we mean, for example, either computationally or information-theoretically, by 'strong enough'.

${ }^{37}$ In this respect my argument can be summed as follows: At the mathematical level, as algebraic structuralists, we are both committed to some type of schema like "If theory T is acceptable (coherent or consistent, for example), then the objects over which it ranges exist". With respect to the acceptability of T, we both agree that all we have is relative consistency (or relative coherence). At the meta-mathematical level, however, as pure algebraic structuralists, the statement of T's acceptability (or consistency) is taken as internally assertory, i.e., is assertory in some stronger theory $\mathrm{T}^{\mathrm{S}}$ which we take as "acceptable" for the purpose of proving the relative consistency of the theory $\mathrm{T}$. The statement ' $\mathrm{T}$ is consistent' holds in $\mathrm{T}^{\mathrm{S}}$, but it is not externally assertory in the sense that we need take this stronger theory $\mathrm{T}^{\mathrm{S}}$ itself as true. Where we differ, then, is that I deny the claim that statements of acceptability (or consistency, coherence etc.,) are externally assertory in the sense that at some point the "If ..., then..." dissolves because the statement of the acceptability of $\mathrm{T}$ is expressed in some true, assertory, meta-mathematical background, theory $\mathrm{T}^{\mathrm{T}}$, that stops the possible regress of stronger theories, i.e., because for some $\mathrm{T}^{\mathrm{S}}, \mathrm{T}^{\mathrm{S}}=\mathrm{T}^{\mathrm{T}}$
} 
Shapiro, to avoid what he sees as a possible infinite regress of using stronger (perhaps, higher-order cardinal) set theories to prove the acceptability (coherence or consistency) of some mathematical theory, holds that we must, at some point, take some one (set-theoretic, modal set-theoretic or structure-theoretic) theory to be true (hence take its axioms/theorems as assertions). But again, the way my picture is painted, Shapiro's move, even if it appeals to some true theory $\mathrm{T}^{\mathrm{T}}$, does not stop the regress. Consider the status of the statement of the truth of this theory $\mathrm{T}^{\mathrm{T}}$ : Where does this statement occur, where and by what means is this statement asserted/proved? Certainly, in light of Gödel, it is not provable in $\mathrm{T}^{\mathrm{T}}$.

Put otherwise, as I stated on page 10, these "acceptability problems" are either equally pointed or they equally dissolve. That is, we can now see the extent to which Shapiro is in the same boat with respect to statements of truth as the pure algebraic structuralist is with respect to statements of consistency. All we need ask is: What of the statement of the truth of your assertory meta-mathematical background theory? Is that statement made in some philosophical theory of truth? If so, then you too need to "turn to philosophy". Is it made in some stronger mathematical theory? If so, then you too are led to a possibly infinite regress of theories. And if he says, contra his own claims, in Shapiro (1991), that $\mathrm{T}^{\mathrm{T}}$ is taken as true because the axioms are, in an Aristotelian sense, "proof-less" or indubitable/irrefutable, then he, like Hilbert, needs to have some "philosophical" story to tell. Otherwise, if he says, now in-line with Shapiro (1991), that he needs no story, but rather can simply claim "I take it as true for such and such foundational reasons", then, likewise, the pure algebraic structuralist, can say "I take it as consistent for such and such foundational reasons", where, again, the category-theoretic structuralist's foundational reasons are taken as organizational as opposed to Aristotelian.

Contra Shapiro, then, we do not have to supplant the rationally reconstructed Hilbertian, and now pure algebraic structuralist, organizational reason by adding a "foundation" as an assertory meta-mathematical background theory. Simply, the mathematical structure of the concepts of the branches of mathematics, either abstractly or set-theoretically organized, and the concept of a category itself, can be conceptually analysed and so mathematically founded by the various category-theoretic axiom systems, i.e., by the EM, ETCS and CCAF axioms, respectively. And the logical structure of the various cat-structured axiom systems can themselves be contentually analysed (either semantically, syntactically or finitistically) and so meta-mathematically founded by means of the various categorical logics

Category theory, then, has as much to say about an pure algebraic consideration of meta-mathematical, or contentual, analyses of logical structure as it does about a algebraic consideration of a mathematical, or conceptual, analyses of mathematical structure, without requiring either an assertory meta-mathematical background theory as a "foundation", and too without turning logical issues into "philosophical" ones. Thus, we can use category theory to frame a pure algebraic interpretation according to which we can be structuralists all the way down.

Open Access This article is distributed under the terms of the Creative Commons Attribution Noncommercial License which permits any noncommercial use, distribution, and reproduction in any medium, provided the original author(s) and source are credited. 


\section{References}

Antonelli, A., \& May, R. (2002). Frege's new science. Notre Dame Journal of Formal Logic, 41(3), 242-270.

Awodey, S. (2004). An answer to Hellman's question: "Does category theory provide a framework for mathematical structuralism?. Philosophia Mathematica, 13(1), 54-64.

Bernays, P. (1967). Hilbert, David. In P. Edwards (Ed.), The encyclopedia of philosophy (Vol. 3, pp. 496-504). New York: Macmillan.

Ewald, W. (1999). From Kant to Hilbert: A source book in the foundations of mathematics (Vol. II). Oxford: Oxford University Press.

Goldfarb, W. D. (1979). Logic in the twenties: The nature of the quantifier. The Journal of Symbolic Logic, 44(3), 351-368.

Hallett, M. (1990). Physicalism, reductionism \& Hilbert. In A. D. Irvine (Ed.), Physicalism in mathematics. Dordretch: Kluwer Academic Publishers.

Hallett, M. (1994). Hilbert's axiomatic method and the laws of thought. In A. George (Ed.), Mathematics and Mind (pp. 158-200). Oxford: Oxford University Press.

Hallett, M. (2007). Reflections on the purity of method in Hilbert's Grundlagen der Geometrie, manuscript.

Hallett, M., \& Majer, U. (2004). David Hilbert's lectures on the foundations of geometry, 1891-1902. New York: Springer Verlag.

Hellman, G. (2003). Does category theory provide a framework for mathematical structuralism. Philosophia Mathematica, 11(2), 129-157.

Hilbert, D. (1899). Grundlagen der Geometrie, Leipzig, Teuber; Foundations of geometry (E. Townsend, 1959, Trans.). La salle, IL: Open Court.

Johnstone, P. T. (2002). Sketches of an elephant: A Topos theory compendium (Vols. 1, 2). Oxford: Oxford University Press.

Kreisel, G. (1960). Ordinal logics and the characterization of informal notions of proof. In J. A. Todd (Ed.), Proceedings of the international congress of mathematicians (pp. 289-299). Cambridge: Cambridge University Press.

Lambek, J., \& Scott, P. J. (1986). Introduction to higher order categorical logic. Cambridge: Cambridge University Press.

Landry, E., \& Marquis, J.-P. (2005). Categories in context: Historical, foundational and philosophical (co-author Jean-Pierre Marquis). Philosophia Mathematica, 13(1), 1-43.

Lawvere, F. W. (1964). An elementary theory of the category of sets. Proceedings of the National Academy of Sciences USA, 52, 1506-1511.

Lawvere, F. W. (1966). The category of categories as a foundation for mathematics. In Proceedings of the conference on categorical algebra (pp. 1-21). La Jolla, New York: Springer-Verlag.

Mac Lane, S. (1968). Foundations of mathematics: Category theory. In R. Klibansky (Ed.), Contemporary philosophy (Vol. I, pp 286-294). Firenze: La Nuova Italia Editrice.

Mac Lane, S. (1986). Mathematics, form and function. New York: Springer-Verlag.

Mac Lane, S. (1996). Structure in mathematics. Philosophia Mathematica, 3(4), 174-183.

Makkai, M., \& Reyes, G. E. (1977). First order categorical logic, Lecture Notes in Math. Vol. 611. Berlin: Springer-Verlag

Marquis, J.-P. (2007). Category theory. In Stanford encyclopedia of philosophy (SEP). http://plato. stanford.edu/entries/category-theory/

McLarty, C. (1991). Axiomatizing a category of categories. Journal of Symbolic Logic, 56(4), 1243-1260.

McLarty, C. (1992). Elementary categories, elementary toposes. Oxford: Oxford University Press.

McLarty, C. (2004). Exploring categorical structuralism. Philosophia Mathematica, 3(1), 37-53.

McLarty, C. (2005). Learning from questions on categorical foundations. Philosophia Mathematica, 13(1), 61-77.

McLarty, C. (2007). The central insight of categorical mathematics. In C. Glymour, W. Wang, \& D. Westerstahl (Eds.), Invited talks of the thirteenth international congress of logic, methodology and philosophy of science, Beijing, 2007. London, King's College Publications (submitted for Studies in Logic and the Foundations of Mathematics)

Parsons, C. (1998). Finitism and intuitive knowledge. In M. Schirn (Ed.), The philosophy of mathematics today (pp. 249-270). Oxford: Oxford University Press.

Shapiro, S. (1991). Foundations without foundationalism. Oxford: Oxford University Press. 
Shapiro, S. (1997). Philosophy of Mathematics: structure and ontology. Oxford: Oxford University Press.

Shapiro, S. (2005). Categories, structures, and the Frege-Hilbert controversy: The status of meta-mathematics. Philosophia Mathematica, 13(1), 61-77.

Tait, W. (1981). Finitism. Journal of Philosophy, 78, 524-546.

Zach, R. (2006). Hilbert's program then and now. In D. Jacquette (Ed.), Handbook of the philosophy of science, Volume 5: Philosophy of Logic. Amsterdam: Elsevier 